Research Article

\title{
A Study on Prevalence of Diabetes Mellitus and the Factors Influencing among the Age Group of 20-60 Years in Urban Population of Mansurabad, Hyderabad, Telangana
}

\author{
D Ravi Babu', G Das Joel ${ }^{2}$, D Subrahmanyam ${ }^{3}$, D Rajeswari ${ }^{4}$ \\ ${ }^{1}$ Professor and Head, ${ }^{3}$ Statistician, Dept. of Community Medicine, KAMSRC, Hyderabad. \\ ${ }^{2}$ Student of KAMSRC, Hyderabad. \\ ${ }^{4}$ Associate Professor of Zoology, Andhra Mahila Sabha College for Women OU Campus, Hyderabad. \\ DOI: https://doi.org/10.24321/2455.7048.201917
}

\section{I $\quad \mathbf{N} \quad \mathbf{F} \quad \mathbf{O}$}

\section{Corresponding Author: \\ D Ravi Babu, Dept. of Community Medicine, KAMSRC, Hyderabad. \\ E-mail Id: \\ ravibabudamu@yahoo.co.in \\ Orcid Id: \\ https://orcid.org/0000-0003-2438-8997 \\ How to cite this article: \\ Babu DR, Joel GD, Subrahmanyam D, Rajeswari D. A Study on Prevalence of Diabetes Mellitus and the Factors Influencing among the Age Group of 20-60 Years in Urban Population of Mansurabad, Hyderabad, Telangana. Epidem Int 2019; 4(4): 1-7.}

Date of Submission: 2019-12-04

Date of Acceptance: 2019-12-24

\section{$\begin{array}{llllllll}\mathbf{A} & \mathbf{B} & \mathbf{S} & \mathbf{T} & \mathbf{R} & \mathbf{A} & \mathbf{C} & \mathbf{T}\end{array}$}

Introduction: Diabetes is a Global Emergency. One in eleven adults have diabetes and one in two adults are with diabetes un diagnosed in 2015.

Objectives: To study the prevalence of diabetes and its factors responsible among the age group of 20 to 60 years in urban area of Mansurabad, in Hyderabad.

Methods: Cross Sectional study was conducted using the semi structure schedule and data was collected from 501 individuals on religion, socioeconomic status, presence of diabetes, habitual consumption of alcohol, habitual smoking, sleep duration per day, presence of stress at home/ work and also collected data on the components of Indian Diabetic Risk Score (IDRS) and later summed to obtain IDRS score $\geq 60$ and $<60$. Logistic regression analyses were performed to determine significant factors influencing diabetes.

Result: Mean age of the population was 40.31 (SD+10.24), females comprised $60 \%$ (301) and males $40 \%$ (200). Socio-economic status score mean was 14.23 (SD+5.75). Eighty-eight (17.56\%) individuals reported having diabetes. Four hundred and one (80\%) reported they did not have diabetes. Twelve (2.39\%) individuals reported they did not know their diabetic status. Approx. $36.52 \%$ (183) had IDRS score $\geq 60$. Habitual consumption of alcohol was noted in $43.71 \%$ (219). Habitual smoking was noted in $26.74 \%$ (134). The duration of sleep less than 6 hrs per day was reported by $42.11 \%$ (211). Stress at work, or home or at both places was reported by $46.9 \%$ (235). Logistic regression analyses (adjusted model) indicated being female (odds ratio 2.23; reference 'male'), IDRS $\geq 60$ (odds ratio 4.26; reference IDRS <60),

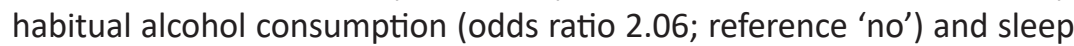
duration $<6 \mathrm{hrs}$. per day (odds ratio 2.07; reference 'sleep duration 6-8 hrs. per day) were significant factors influencing diabetes.

Conclusion: Educational measures should aim at encouraging regular physical exercise, refraining from alcohol consumption and maintaining adequate sleep duration of 6-8 hrs per day.

Keywords: Indian Diabetic Risk Score, diabetes, Waist Circumference, Lifestyle Disorder 


\section{Introduction}

Diabetes mellitus is a pandemic in the world. The pathological complications associated with diabetes are increasing morbidity and mortality. ${ }^{1}$ Worldwide about 382 million people are living with Diabetes Mellitus as reported by International Diabetes Federation (IDF) in $2013 .{ }^{2} \mathrm{An}$ alarmingly high proportion of them about 175 million $(46 \%)$ are yet undiagnosed and are progressing towards complications. IDF statistics predicts a $55 \%$ increase in global diabetes burden by 2035. It used to be disease of affluent and developed nations. But, most distressing about diabetes mellitus is that it is now appearing in the younger generation and low- and middle-income group of people where number of people is more. Studies are showing that it may be due to change in their lifestyle especially in the age group of 20-79 years.

In India 65.1 million people in the age group of 20-79 years are Diabetics Mellitus and this figure is predicted to increase to 79.4 million by 2030. ${ }^{3,4}$ According to National Urban Diabetes Survey (NUDS) prevalence of diabetes in Southern parts of India is $16.6 \%$ in Hyderabad, $13.5 \%$ in Chennai, $12.4 \%$ in Bengaluru, $11.7 \%$ in Kolkata, $11.6 \%$ in New Delhi and 9.3\% in Mumbai. ${ }^{5}$

Due to heavy burden of diabetes, simple tools should be devised to screen the problem in apparently healthy community. With this objective in the current study, a simple and cost-effective screening tool such as Indian Diabetic Risk Score (using four simple parameters viz., age, abdominal obesity, family history of diabetes, and physical activity) developed by Mohan et al, (2005) ${ }^{6}$ was used. We have planned a study Using IDRS.

To know the prevalence of diabetes mellitus (type-2) in the age group of 20 to 60 years in the urban population and factors influencing on diabetes mellitus. To know the factors, influence on diabetes mellitus among the age group of 20 to 60 years in Urban population. To suggest prevention measures through Health Education.

\section{Materials and Methods}

A community-based cross-sectional study was conducted in urban area of Mansurabad, Hyderabad, Telangana State, India, which is a field practice area of Urban Health Training Centre (UHTC) of the Department of Community Medicine. Kamineni Academy of Medical Sciences and Research Centre Hyderabad. The study was conducted from May to July 2016. Sample size was arrived by using the method mentioned by Daniel (1999)' ${ }^{7}$ sing formula $\mathrm{N}=\mathrm{Z} 2 \mathrm{P}(1-\mathrm{P}) / \mathrm{d}^{2}$, where $\mathrm{N}=$ sample size, $\mathrm{Z}=\mathrm{Z}$ statistic for level of confidence, $\mathrm{P}=$ expected prevalence or proportion and $\mathrm{d}=$ precision. We used $95 \%$ confidence level, $Z$ statistic at this level is 1.96. Expected prevalence was taken as $17 \%$ (according to National Urban Diabetic Survey in Southern parts of
India). ${ }^{5}$ ' $d$ ' is $5 \% . \mathrm{N}=217$. However, the study population included 501 individuals aged 20 to 60 years.

The Age group 20 to 60 yrs was chosen as they are more likely to develop and/or have type 2 Diabetes Mellitus. We have excluded pregnant mothers, individuals with medical condition of distended abdomen from the study. Informed consent was obtained from all study participants and data confidentiality was ensured. We conducted a house to house survey and used a pre-tested semi-structured questionnaire (schedule) to collect data on demographic (age, gender, religion, socio-economic status), Indian Diabetic Risk Score developed by Mohan V. et al. (2005) ${ }^{6}$, diabetic status, and lifestyle characteristics (habitual alcohol consumption, habitual smoking, sleep duration per day, and presence of stress at home, or at work place or both). IDRS was calculated according to the risk scores presented in table 1. The waist circumference was measured according the WHO \& NIH protocol (Figure 1).

Table I.Indian Diabetes Risk Score

\begin{tabular}{|c|c|}
\hline Particulars & Score \\
\hline \multicolumn{2}{|l|}{ Age } \\
\hline$>35$ & 0 \\
\hline $35-49$ & 20 \\
\hline$>50$ & 30 \\
\hline \multicolumn{2}{|l|}{ Waist circumference } \\
\hline Waist < $80 \mathrm{~cm}$ (female), <90 cm (male) & 0 \\
\hline Waist $>80-89 \mathrm{~cm}$ (female), $<09-99 \mathrm{~cm}$ (male) & 10 \\
\hline Waist $\geq 90 \mathrm{~cm}$ (female), $\geq 100 \mathrm{~cm}$ (male) & 20 \\
\hline \multicolumn{2}{|l|}{ Physical activity: } \\
\hline $\begin{array}{l}\text { Regular vigorous exercise or strenuous } \\
\text { (manual) activities at home/ work }\end{array}$ & 0 \\
\hline $\begin{array}{l}\text { Regular moderate exercise or moderate } \\
\text { physical activity at home/ work }\end{array}$ & 10 \\
\hline $\begin{array}{c}\text { Regular mild exercise or mild physical } \\
\text { activity at home/ work }\end{array}$ & 20 \\
\hline $\begin{array}{l}\text { No exercise and/or sedentary activities at } \\
\text { home/ work }\end{array}$ & 30 \\
\hline \multicolumn{2}{|l|}{ Family history of diabetes: } \\
\hline No diabetes in parents & 0 \\
\hline One parent is diabetic & 10 \\
\hline Both parents are diabetic & 20 \\
\hline
\end{tabular}

Minimum Score: 0, Maximum Score: 100, Positive Score (high risk category): $\geq 60 / 100$. Waist circumference was measured with a non-stretchable tape to nearest 0.1 Centimeter $(\mathrm{cm})$ at the midpoint between the lowest rib and the highest point of iliac crest at the end of expiration, according to World Health Organization. 


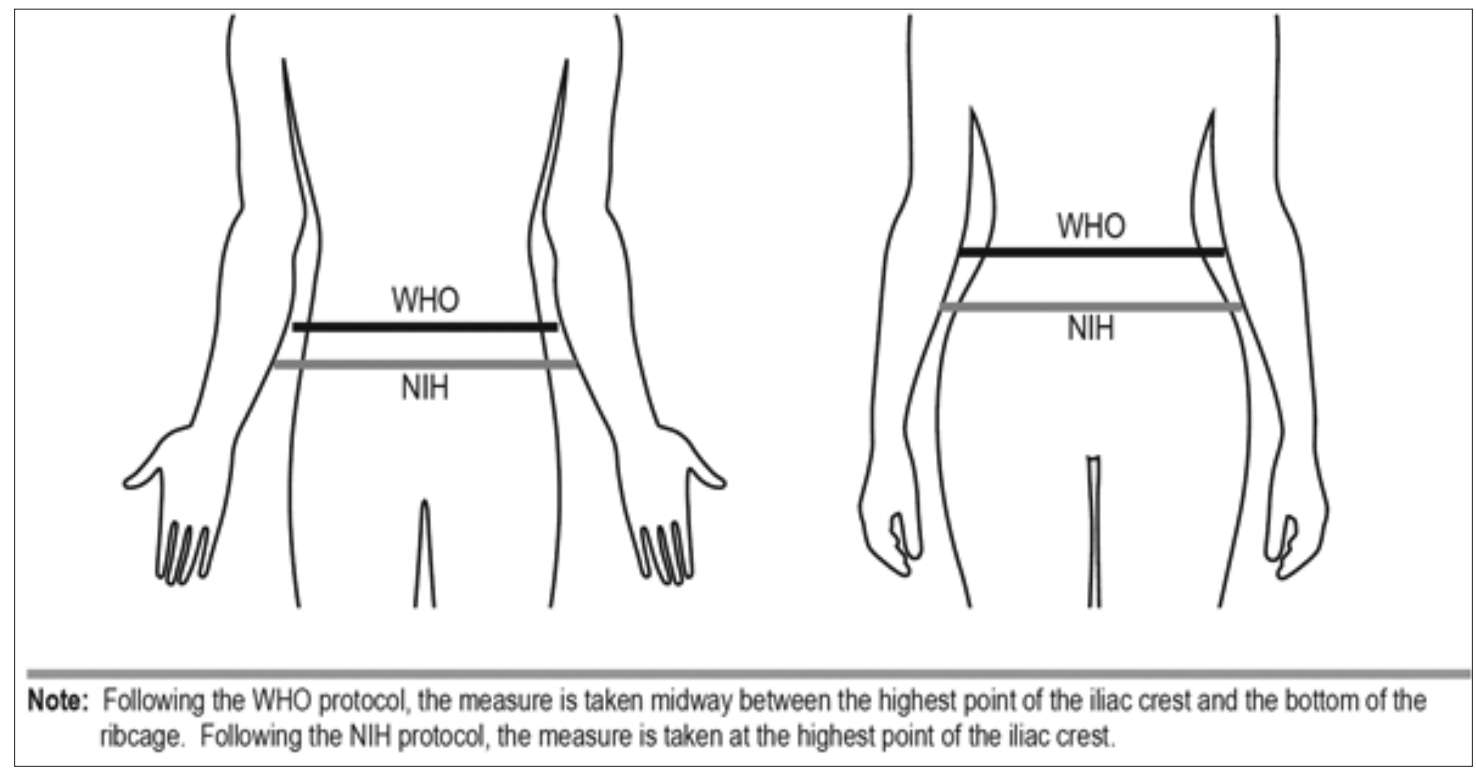

Figure I. Waist circumference measurement sites for men and women based on World Health Organization (WHO) and National Institutes of Health (NIH) protocols

Socio-economic status was calculated according to Kuppuswamy classification. Data was entered in Microsoft Excel Software, statistical analysis software STATA v.11.2 was used for performing data management and statistical analysis. Diabetes status was recorded "Yes, No and don't know." Indian Diabetic Risk Score (IDRS) was categorized "greater than or equal to $60(\geq 60)$ and less than $60(<60)$." Habitual alcohol consumption status was recorded "Yes, No, Refused." Habitual smoking status was recorded "Yes, No." Sleep duration per day was recorded in three categories viz., Less Sleep (<6 hrs. per day), Normal Sleep (6-8 hrs. per day), More Sleep $>9$ hrs. per day. Presence of Stress in their day to day activities at home and/ or at work place or at both places is recorded as "Yes, No."

\section{Result}

Mean age of the study population (501 respondents) was 40.31 yrs. (SD+10.24) Age group 35 to $39 \mathrm{yrs}$. had the highest number of respondents 121 (24.15\%). females comprised $60 \%$, and males $40 \%$ in the study population. Religious composition of the study population was Hindus (88.2\%), Christians (8.8\%) and Muslims (3.0\%). Socio-economic status score mean was 14.23 (SD+5.75) (Table 2). This SES score corresponds to lower middle-class group according to Kuppuswamy classification. Majority of the population was in upper middle class (39.3), lower middle class (28.3) and upper lower class (30.5\%).

Table 2.Demographic and lifestyle characteristics of study population

\begin{tabular}{|c|c|c|c|c|c|c|c|c|c|c|}
\hline Age Groups (Yrs.) & $20-24$ & $25-29$ & $30-34$ & $35-39$ & $40-44$ & $45-49$ & $50-54$ & $55-59$ & 60 & Total \\
\hline N0 (\%) & $\begin{array}{c}28 \\
(5.5)\end{array}$ & $\begin{array}{c}66 \\
(13.17)\end{array}$ & $\begin{array}{c}46 \\
(9.18)\end{array}$ & $\begin{array}{c}121 \\
(24.15)\end{array}$ & $\begin{array}{c}42 \\
(8.38)\end{array}$ & $\begin{array}{c}76 \\
(15.16)\end{array}$ & $\begin{array}{c}61 \\
(12.17)\end{array}$ & $\begin{array}{c}57 \\
(11.37)\end{array}$ & $\begin{array}{c}4 \\
(0.79)\end{array}$ & $\begin{array}{c}501 \\
(100)\end{array}$ \\
\hline \multicolumn{11}{|l|}{ Gender } \\
\hline Female (\%) & $\begin{array}{c}15 \\
(4.98)\end{array}$ & $\begin{array}{c}34 \\
(11.29) \\
\end{array}$ & $\begin{array}{c}25 \\
(8.30) \\
\end{array}$ & $\begin{array}{c}89 \\
(29.56) \\
\end{array}$ & $\begin{array}{c}27 \\
(8.97) \\
\end{array}$ & $\begin{array}{c}45 \\
(14.95)\end{array}$ & $\begin{array}{c}43 \\
(14.28)\end{array}$ & $\begin{array}{c}21 \\
(6.97)\end{array}$ & $\begin{array}{c}2 \\
(0.66)\end{array}$ & $\begin{array}{l}301 \\
(60) \\
\end{array}$ \\
\hline Male (\%) & $\begin{array}{c}13 \\
(6.5)\end{array}$ & $\begin{array}{c}32 \\
(16.0)\end{array}$ & $\begin{array}{c}21 \\
(10.5)\end{array}$ & $\begin{array}{c}32 \\
(16.0)\end{array}$ & $15(7.5)$ & $31(15.5)$ & $\begin{array}{c}18 \\
(9.0)\end{array}$ & $\begin{array}{c}36 \\
(18.0)\end{array}$ & $2(1.0)$ & $\begin{array}{l}200 \\
(40)\end{array}$ \\
\hline SES Mean (SD) & $\begin{array}{l}14.42 \\
(6.15)\end{array}$ & $\begin{array}{l}15.90 \\
(5.33)\end{array}$ & $\begin{array}{l}14.76 \\
(6.15)\end{array}$ & $\begin{array}{l}14.72 \\
(5.17)\end{array}$ & $\begin{array}{c}14.0 \\
(5.69)\end{array}$ & $\begin{array}{l}13.52 \\
(5.15) \\
\end{array}$ & $\begin{array}{l}11.85 \\
(6.13)\end{array}$ & $\begin{array}{l}14.59 \\
(6.47)\end{array}$ & $\begin{array}{l}11.25 \\
(7.27)\end{array}$ & $\begin{array}{l}14.23 \\
(5.75)\end{array}$ \\
\hline \multicolumn{11}{|l|}{ Diabetes } \\
\hline Yes (\%) & - & $4(4.54)$ & $\begin{array}{c}3 \\
(3.40) \\
\end{array}$ & $\begin{array}{c}25 \\
(28.4) \\
\end{array}$ & $3(3.40)$ & $\begin{array}{c}21 \\
(23.86) \\
\end{array}$ & $\begin{array}{c}15 \\
(17.04) \\
\end{array}$ & $\begin{array}{c}15 \\
(17.04) \\
\end{array}$ & $\begin{array}{c}2 \\
(2.27) \\
\end{array}$ & $\begin{array}{c}88 \\
(17.56) \\
\end{array}$ \\
\hline No (\%) & $\begin{array}{c}28 \\
(6.98)\end{array}$ & $\begin{array}{c}57 \\
(14.21)\end{array}$ & $\begin{array}{c}43 \\
(10.72)\end{array}$ & $\begin{array}{c}95 \\
(23.69)\end{array}$ & $\begin{array}{c}37 \\
(9.22)\end{array}$ & $\begin{array}{c}54 \\
(13.46)\end{array}$ & $\begin{array}{c}45 \\
(11.22)\end{array}$ & $\begin{array}{c}40 \\
(9.97)\end{array}$ & $\begin{array}{c}2 \\
(0.49)\end{array}$ & $\begin{array}{c}401 \\
(80.0)\end{array}$ \\
\hline
\end{tabular}




\begin{tabular}{|c|c|c|c|c|c|c|c|c|c|c|}
\hline Don't now (\%) & - & $\begin{array}{c}5 \\
(41.66)\end{array}$ & - & $1(8.33)$ & $\begin{array}{c}2 \\
(16.66)\end{array}$ & $1(8.33)$ & $\begin{array}{c}1 \\
(8.33)\end{array}$ & $\begin{array}{c}2 \\
(16.66)\end{array}$ & - & $\begin{array}{c}12 \\
(2.39)\end{array}$ \\
\hline \multicolumn{11}{|l|}{ IDRS } \\
\hline$>60(\%)$ & - & $1(0.54)$ & $\begin{array}{c}1 \\
(0.54)\end{array}$ & $\begin{array}{c}46 \\
(25.13)\end{array}$ & $\begin{array}{c}11 \\
(6.01)\end{array}$ & $\begin{array}{c}30 \\
(16.39)\end{array}$ & $\begin{array}{c}47 \\
(25.68)\end{array}$ & $\begin{array}{c}44 \\
(24.04)\end{array}$ & $\begin{array}{c}3 \\
(1.63)\end{array}$ & $\begin{array}{c}183 \\
(36.52)\end{array}$ \\
\hline$<60(\%)$ & $\begin{array}{c}28 \\
(8.80) \\
\end{array}$ & $\begin{array}{c}65 \\
(20.44) \\
\end{array}$ & $\begin{array}{c}45 \\
(14.15) \\
\end{array}$ & $\begin{array}{c}75 \\
(23.58) \\
\end{array}$ & $\begin{array}{c}31 \\
(9.74) \\
\end{array}$ & $\begin{array}{c}46 \\
(14.46) \\
\end{array}$ & $\begin{array}{c}14 \\
(4.40) \\
\end{array}$ & $\begin{array}{c}13 \\
(7.23) \\
\end{array}$ & $\begin{array}{c}1 \\
(0.31)\end{array}$ & $\begin{array}{c}318 \\
(63.47) \\
\end{array}$ \\
\hline \multicolumn{11}{|l|}{ Alcohol } \\
\hline Yes (\%) & $\begin{array}{c}7 \\
(3.19) \\
\end{array}$ & $\begin{array}{c}23 \\
(10.50) \\
\end{array}$ & $\begin{array}{c}19 \\
(8.67) \\
\end{array}$ & $\begin{array}{c}49 \\
(22.37) \\
\end{array}$ & $\begin{array}{c}18 \\
(8.21) \\
\end{array}$ & $\begin{array}{c}36 \\
(16.43) \\
\end{array}$ & $\begin{array}{c}28 \\
(12.78) \\
\end{array}$ & $\begin{array}{c}37 \\
(16.89) \\
\end{array}$ & $\begin{array}{c}2 \\
(0.91) \\
\end{array}$ & $\begin{array}{c}219 \\
(43.71) \\
\end{array}$ \\
\hline No (\%) & $\begin{array}{c}21 \\
(8.20) \\
\end{array}$ & $\begin{array}{c}41 \\
(16.01)\end{array}$ & $\begin{array}{c}27 \\
(10.54) \\
\end{array}$ & $\begin{array}{c}61 \\
(23.82) \\
\end{array}$ & $\begin{array}{c}23 \\
(8.98) \\
\end{array}$ & $\begin{array}{c}35 \\
(13.67) \\
\end{array}$ & $\begin{array}{c}27 \\
(10.54) \\
\end{array}$ & $\begin{array}{c}19 \\
(7.42) \\
\end{array}$ & $\begin{array}{c}2 \\
(0.78)\end{array}$ & $\begin{array}{c}256 \\
(51.09) \\
\end{array}$ \\
\hline Refused (\%) & - & $1(4.0)$ & - & $\begin{array}{c}11 \\
(44.0)\end{array}$ & $1(4.0)$ & $5(20.0)$ & $\begin{array}{c}6 \\
(24.0) \\
\end{array}$ & $1(4.0)$ & - & $\begin{array}{c}25 \\
(4.99) \\
\end{array}$ \\
\hline \multicolumn{11}{|l|}{ Smoking } \\
\hline Yes (\%) & $\begin{array}{c}4 \\
(2.98) \\
\end{array}$ & $\begin{array}{c}11 \\
(8.20) \\
\end{array}$ & $\begin{array}{c}2 \\
(1.49) \\
\end{array}$ & $\begin{array}{c}37 \\
(27.61) \\
\end{array}$ & $8(5.97)$ & $\begin{array}{c}29 \\
(21.64) \\
\end{array}$ & $\begin{array}{c}20 \\
(14.92) \\
\end{array}$ & $\begin{array}{c}22 \\
(16.41) \\
\end{array}$ & $\begin{array}{c}1 \\
(0.74)\end{array}$ & $\begin{array}{c}134 \\
(26.74) \\
\end{array}$ \\
\hline No (\%) & $\begin{array}{c}24 \\
(6.55) \\
\end{array}$ & $\begin{array}{c}54 \\
(14.75) \\
\end{array}$ & $\begin{array}{c}44 \\
(12.02) \\
\end{array}$ & $\begin{array}{c}84 \\
(22.95) \\
\end{array}$ & $\begin{array}{c}34 \\
(9.28) \\
\end{array}$ & $\begin{array}{c}47 \\
(12.84) \\
\end{array}$ & $\begin{array}{c}41 \\
(11.20) \\
\end{array}$ & $\begin{array}{c}34 \\
(9.28) \\
\end{array}$ & $\begin{array}{c}3 \\
(0.81)\end{array}$ & $\begin{array}{c}366 \\
(73.05) \\
\end{array}$ \\
\hline \multicolumn{11}{|l|}{ Sleep Duration } \\
\hline$<6$ hrs. (\%) & $\begin{array}{c}5 \\
(2.36) \\
\end{array}$ & $\begin{array}{c}12 \\
(5.68)\end{array}$ & $\begin{array}{c}10 \\
(4.73) \\
\end{array}$ & $\begin{array}{c}67 \\
(31.75) \\
\end{array}$ & $\begin{array}{c}18 \\
(8.53)\end{array}$ & $\begin{array}{c}41 \\
(19.43) \\
\end{array}$ & $\begin{array}{c}30 \\
(14.21) \\
\end{array}$ & $\begin{array}{c}26 \\
(12.32) \\
\end{array}$ & $\begin{array}{c}2 \\
(0.94) \\
\end{array}$ & $\begin{array}{c}211 \\
(42.11) \\
\end{array}$ \\
\hline 6-8 hrs. (\%) & $\begin{array}{c}21 \\
(8.23) \\
\end{array}$ & $\begin{array}{c}45 \\
(17.64) \\
\end{array}$ & $\begin{array}{c}35 \\
(13.72) \\
\end{array}$ & $\begin{array}{c}47 \\
(18.43) \\
\end{array}$ & $\begin{array}{c}20 \\
(7.84) \\
\end{array}$ & $\begin{array}{c}31 \\
(12.15) \\
\end{array}$ & $\begin{array}{c}28 \\
(10.98) \\
\end{array}$ & $\begin{array}{c}27 \\
(10.58) \\
\end{array}$ & $\begin{array}{c}1 \\
(0.39) \\
\end{array}$ & $\begin{array}{c}255 \\
(50.89) \\
\end{array}$ \\
\hline$>9 \mathrm{hrs}$. & $\begin{array}{c}2 \\
(5.88)\end{array}$ & $\begin{array}{c}8 \\
(23.52)\end{array}$ & $\begin{array}{c}1 \\
(2.94)\end{array}$ & $\begin{array}{c}7 \\
(20.58)\end{array}$ & $\begin{array}{c}4 \\
(11.76)\end{array}$ & $4(11.76)$ & $\begin{array}{c}3 \\
(8.82)\end{array}$ & $\begin{array}{c}4 \\
(11.76)\end{array}$ & $\begin{array}{c}1 \\
(2.94)\end{array}$ & $\begin{array}{c}34 \\
(6.78)\end{array}$ \\
\hline \multicolumn{11}{|l|}{ Stress } \\
\hline Yes (\%) & $\begin{array}{c}3 \\
(1.27)\end{array}$ & $\begin{array}{c}17 \\
(7.23)\end{array}$ & $\begin{array}{c}19 \\
(8.08)\end{array}$ & $\begin{array}{c}65 \\
(27.65)\end{array}$ & $\begin{array}{c}21 \\
(8.93)\end{array}$ & $\begin{array}{c}43 \\
(18.29) \\
\end{array}$ & $\begin{array}{c}33 \\
(14.04)\end{array}$ & $\begin{array}{c}34 \\
(14.46)\end{array}$ & - & $\begin{array}{c}235 \\
(46.90)\end{array}$ \\
\hline No (\%) & $\begin{array}{c}25 \\
(9.43)\end{array}$ & $\begin{array}{c}48 \\
(18.11)\end{array}$ & $\begin{array}{c}27 \\
(10.18)\end{array}$ & $\begin{array}{c}56 \\
(21.13)\end{array}$ & $\begin{array}{c}21 \\
(7.92)\end{array}$ & $\begin{array}{c}33 \\
(12.45)\end{array}$ & $\begin{array}{c}28 \\
(10.56)\end{array}$ & $\begin{array}{c}23 \\
(8.67)\end{array}$ & $\begin{array}{c}4 \\
(1.50)\end{array}$ & $\begin{array}{c}265 \\
(52.89)\end{array}$ \\
\hline
\end{tabular}

Table 3.Adjusted and Unadjusted Odds Ratios for diabetes based on demographic and lifestyle characteristics

\begin{tabular}{|c|c|c|c|c|c|c|}
\hline Characteristic & Unadjusted OR & $P>|z|$ & $95 \% \mathrm{Cl}$ & Adjusted OR & $P>|z|$ & $95 \% \mathrm{Cl}$ \\
\hline Age & 1.06 & $0.000^{*}$ & 1.035 to 1.087 & 1.01 & 0.348 & 0.982 to 1.051 \\
\hline $\begin{array}{l}\text { Gender } \\
\text { Female } \\
\text { (ref. Male) }\end{array}$ & $\begin{array}{c}2.44 \\
1.0\end{array}$ & $0.001^{*}$ & 1.441 to 4.148 & $\begin{array}{c}2.23 \\
1.0\end{array}$ & $0.023^{*}$ & 1.118 to 4.447 \\
\hline SES score & 0.96 & 0.078 & 0.924 to 1.004 & 0.99 & 0.794 & 0.944 to 1.044 \\
\hline $\begin{array}{c}\text { IDRS } \\
>60 \\
\text { (ref. }<60)\end{array}$ & $\begin{array}{c}7.20 \\
1.0\end{array}$ & $0.000^{*}$ & 4.269 to 12.150 & $\begin{array}{c}4.26 \\
1.0 \\
\end{array}$ & $0.000^{*}$ & 2.247 to 8.110 \\
\hline $\begin{array}{c}\text { Smoking Yes } \\
\text { (ref. No) }\end{array}$ & $\begin{array}{c}3.33 \\
1.0 \\
\end{array}$ & $0.000^{*}$ & 2.066 to 5.395 & $\begin{array}{c}1.55 \\
1.0 \\
\end{array}$ & 0.171 & 0.826 to 2.927 \\
\hline $\begin{array}{l}\text { Alcohol } \\
\text { Refused Yes } \\
\text { (ref. No) }\end{array}$ & $\begin{array}{c}8.23 \text { to } 2.77 \\
1.0\end{array}$ & $\begin{array}{l}0.000^{*} \\
0.000^{*}\end{array}$ & $\begin{array}{l}3.391 \text { to } 19.987 \\
1.650 \text { to } 4.662\end{array}$ & $\begin{array}{l}3.12 \\
2.06\end{array}$ & $\begin{array}{l}0.036^{*} \\
0.029^{*}\end{array}$ & $\begin{array}{l}1.077 \text { to } 9.083 \\
1.075 \text { to } 3.957\end{array}$ \\
\hline
\end{tabular}




\begin{tabular}{|c|c|c|c|c|c|c|}
\hline $\begin{array}{l}\text { Stress Yes } \\
\text { (ref. No) }\end{array}$ & $\begin{array}{c}2.64 \\
1.0\end{array}$ & $0.000^{*}$ & 1.629 to 4.285 & $\begin{array}{c}1.16 \\
1.0\end{array}$ & 0.610 & 0.641 to 2.132 \\
\hline $\begin{array}{c}\text { Sleep }<6 \text { hrs. } \\
>9 \text { hrs. } \\
\text { (ref. 6-8 hrs.) }\end{array}$ & $\begin{array}{c}4.96 \\
0.34 \\
1.0\end{array}$ & $\begin{array}{c}0.000^{*} \\
0.310\end{array}$ & $\begin{array}{l}2.912 \text { to } 8.471 \\
0.045 \text { to } 2.672\end{array}$ & $\begin{array}{c}2.07 \\
0.38 \\
1.0\end{array}$ & $\begin{array}{c}0.027^{*} \\
0.365\end{array}$ & $\begin{array}{l}1.085 \text { to } 3.964 \\
0.048 \text { to } 3.053\end{array}$ \\
\hline
\end{tabular}

In this study population $17.56 \%$ ( 88 respondents) indicated they were suffering from diabetes. Out of 401 individuals, about $80 \%$ them said that they are not suffering from diabetes. Only $2.39 \%$ (12 respondents) indicated they did not know their diabetic status. IDRS score $\geq 60$ was observed in $36.52 \%$ (183 respondents). The distribution of diabetes among those with IDRS score greater than or equal to i.e., $\geq 60$ and less than $60(<60)$ among males and females grouped by age is presented in Table 4 . Among those with IDRS score $\geq 60$, in females, approx. $43 \%$ (52 out of 122 female respondents) indicated they were diabetic. Similarly, in males, approx. 21\% (13 out of 61 male respondents) indicated they were diabetic. Among those with IDRS score $<60$, in females, approx. $8 \%$ (15 out of 179 female respondents) indicated they were diabetic. Similarly, in males approx. 6\% (8 out of 139 male respondents) were diabetic. Regular alcohol consumption was noted in $43.71 \%$ ( 219 respondents). About $5 \%$ refused to respond to their alcohol consumption status. Habitual smoking was identified among $26.74 \%$ (134 respondents). Normal sleep duration of 6-8 hours per day was observed in $50.89 \%$ ( 255 respondents). Fewer than 6 hrs. per day Sleep duration was noted among $42.11 \%$ ( 211 respondents). Approximately $47 \%$ ( 235 respondents) reported high stress levels at either their home, work or at both places.
Logistic regression analyses were performed to identify significant contributing factors to diabetes outcome among 501 respondents in the age group 20 to $60 \mathrm{yrs}$. Adjusted and unadjusted Odds Ratios (OR) along with Confidence Intervals $(\mathrm{Cl})$ and $\mathrm{p}$-values are presented in Table 3.

Four factors-Gender (Females), IDRS score $\geq 60$, Alcohol consumption (Yes), and less than normal sleep duration ( $<6$ hrs. per day) were found be significantly influencing diabetes in both adjusted and unadjusted logistic regression models. Individuals with IDRS $\geq 60$ had an adjusted Odds Ratio (OR) 4.26 (95\% Cl: 2.247 to 8.110, p-value <0.001). The unadjusted OR for IDRS >60 was 7.20 (95\% Cl: 4.269 to $12.15, \mathrm{p}$ value $<0.001)$. For females the adjusted $\mathrm{OR}$ was 2.23 (95\% Cl: 1.118 to $4.447 \mathrm{p}$-values 0.023$)$. The unadjusted OR was 2.44 (95\% Cl: 1.441 to $4.148 \mathrm{p}$-value 0.001 ) The adjusted OR for Alcohol consumption was 2.06 ( $95 \%$ Cl: 1.075 to 3.957 , p-value 0.029). The unadjusted OR for alcohol consumption was 2.77 ( $95 \% \mathrm{Cl}: 1.650$ to 4.662 , $\mathrm{p}$-value $<0.001)$. Individuals with sleep duration less than 6 hrs. per day had an adjusted OR of 2.07 (95\% Cl: 1.085 to $3.964, p$-value 0.027 ). The unadjusted $O R$ for sleep duration less than 6 hrs. per day is 4.96 (95\% Cl: 2.912 to $8.471 \mathrm{p}$-value $<0.001$ ). Age, Socio-economic status score, habitual smoking, Stress were not found to be significantly influencing diabetes in adjusted models (Table 3 ).

Table 4.Distribution of Diabetes in IDRS $\geq 60$ and IDRS $<60$ in relation to age groups and gender

\begin{tabular}{|c|c|c|c|c|c|c|c|c|c|c|c|c|}
\hline & \multicolumn{4}{|c|}{ Diabetes } & \multicolumn{4}{|c|}{ Diabetes } & \multicolumn{4}{|c|}{ Diabetes } \\
\hline & \multicolumn{4}{|c|}{ IDRS >60 } & \multicolumn{4}{|c|}{ IDRS $<60$} & \multicolumn{4}{|c|}{ IDRS combined } \\
\hline & $\begin{array}{l}\text { Don't } \\
\text { know }\end{array}$ & No & Yes & Total & $\begin{array}{l}\text { Don't } \\
\text { know }\end{array}$ & No & Yes & Total & $\begin{array}{l}\text { Don't } \\
\text { know }\end{array}$ & No & Yes & Total \\
\hline Female & & & & & & & & & & & & \\
\hline $20-24$ & 0 & 0 & 0 & 0 & 0 & 15 & 0 & 15 & 0 & 15 & 0 & 15 \\
\hline $25-29$ & 0 & 1 & 0 & 1 & 3 & 26 & 4 & 33 & 3 & 27 & 4 & 34 \\
\hline $30-34$ & 0 & 0 & 0 & 0 & 0 & 24 & 1 & 25 & 0 & 24 & 1 & 25 \\
\hline $35-39$ & 1 & 19 & 18 & 38 & 0 & 48 & 3 & 51 & 1 & 67 & 21 & 89 \\
\hline $40-44$ & 1 & 4 & 2 & 7 & 0 & 19 & 1 & 20 & 1 & 23 & 3 & 27 \\
\hline $45-49$ & 1 & 10 & 10 & 21 & 0 & 18 & 6 & 24 & 1 & 28 & 16 & 45 \\
\hline $50-54$ & 1 & 21 & 13 & 35 & 0 & 8 & 0 & 8 & 1 & 29 & 13 & 43 \\
\hline $55-59$ & 0 & 10 & 8 & 18 & 0 & 3 & 0 & 3 & 0 & 13 & 8 & 21 \\
\hline 60 & 0 & 1 & 1 & 2 & 0 & 0 & 0 & 0 & 0 & 1 & 1 & 2 \\
\hline $\begin{array}{l}\text { Total } \\
\text { (\%) }\end{array}$ & $\begin{array}{c}4 \\
(3.2)\end{array}$ & $\begin{array}{c}66 \\
(54.0)\end{array}$ & $\begin{array}{c}52 \\
(42.6)\end{array}$ & $\begin{array}{c}122 \\
(100)\end{array}$ & $\begin{array}{c}3 \\
(1.67)\end{array}$ & $\begin{array}{c}161 \\
(89.9)\end{array}$ & $\begin{array}{c}15 \\
(8.37)\end{array}$ & $\begin{array}{c}179 \\
(100)\end{array}$ & $\begin{array}{c}7 \\
(2.35)\end{array}$ & $\begin{array}{c}227 \\
(75.41)\end{array}$ & $\begin{array}{c}67 \\
(22.25)\end{array}$ & $\begin{array}{c}301 \\
(100)\end{array}$ \\
\hline
\end{tabular}




\begin{tabular}{|c|c|c|c|c|c|c|c|c|c|c|c|c|}
\hline Male & & & & & & & & & & & & \\
\hline $20-24$ & 0 & 0 & 0 & 0 & 0 & 13 & 0 & 13 & 0 & 0 & 13 & 13 \\
\hline $25-29$ & 0 & 0 & 0 & 0 & 2 & 30 & 0 & 32 & 2 & 30 & 0 & 32 \\
\hline $30-34$ & 0 & 1 & 0 & 1 & 0 & 18 & 2 & 20 & 0 & 19 & 2 & 21 \\
\hline $35-39$ & 0 & 6 & 2 & 8 & 0 & 22 & 2 & 24 & 0 & 28 & 4 & 32 \\
\hline $40-44$ & 1 & 3 & 0 & 4 & 0 & 11 & 0 & 11 & 1 & 14 & 0 & 15 \\
\hline $45-49$ & 0 & 7 & 2 & 9 & 0 & 19 & 3 & 22 & 0 & 26 & 5 & 31 \\
\hline $50-54$ & 0 & 10 & 2 & 12 & 0 & 6 & 0 & 6 & 0 & 16 & 2 & 18 \\
\hline 55-59 & 0 & 20 & 6 & 26 & 2 & 7 & 1 & 10 & 2 & 27 & 7 & 36 \\
\hline 60 & 0 & 0 & 1 & 1 & 0 & 1 & 0 & 1 & 0 & 1 & 1 & 2 \\
\hline $\begin{array}{c}\text { Column } \\
\text { Total } \\
(\%)\end{array}$ & $\begin{array}{c}1 \\
(1.63)\end{array}$ & $\begin{array}{c}47 \\
(77.04)\end{array}$ & $\begin{array}{c}13 \\
(21.31)\end{array}$ & $\begin{array}{c}61 \\
(100)\end{array}$ & $\begin{array}{c}4 \\
(2.87)\end{array}$ & $\begin{array}{c}127 \\
(91.36)\end{array}$ & $\begin{array}{c}8 \\
(5.75)\end{array}$ & $\begin{array}{c}139 \\
(100)\end{array}$ & $\begin{array}{c}5 \\
(2.5)\end{array}$ & $\begin{array}{c}174 \\
(87.0)\end{array}$ & $\begin{array}{c}21 \\
(10.5)\end{array}$ & $\begin{array}{c}200 \\
(100)\end{array}$ \\
\hline $\begin{array}{c}\text { Grand } \\
\text { Total } \\
\text { (\%) }\end{array}$ & $\begin{array}{c}5 \\
(2.73)\end{array}$ & $\begin{array}{c}113 \\
(61.74)\end{array}$ & $\begin{array}{c}65 \\
(35.5)\end{array}$ & $\begin{array}{c}183 \\
(100)\end{array}$ & $\begin{array}{c}7 \\
(2.2)\end{array}$ & $\begin{array}{c}288 \\
(90.56)\end{array}$ & $\begin{array}{c}23 \\
(7.23)\end{array}$ & $\begin{array}{c}318 \\
(100)\end{array}$ & $\begin{array}{c}12 \\
(2.39)\end{array}$ & $\begin{array}{c}401 \\
(80.03)\end{array}$ & $\begin{array}{c}88 \\
(17.56)\end{array}$ & $\begin{array}{c}501 \\
(100)\end{array}$ \\
\hline
\end{tabular}

\section{Discussion}

In this study, respondents in IDRS $\geq 60$ comprised $36.52 \%$ (183 respondents). In a similar study conducted by Suraj et al., $(2015)^{8}$ in urban area of Jamnagar city $22.8 \%$ (114 respondents) were categorized in IDRS $\geq 60$. The distribution of population in high risk category (IDRS $\geq 60$ ) was higher $(59 \%)$ in the study conducted by Geetha M et al. $(2014)^{2}$ and lower (31.5\%) in the study done by Chowdhury R et al. (2012). ${ }^{9}$

In this study, distribution of IDRS $\geq 60$ (high risk group) in age group $<35$ yrs. was $1.4 \%$ ( 2 respondents), 35-49 yrs. age group was $36.4 \%$ ( 87 respondents), $>50$ yrs. were $77 \%$ (94 respondents). Whereas in a study by Suraj et al. $(2015)^{8}$ in urban area of Jamnagar city $<35$ yrs. was $0 \%$ (0), $35-49$ yrs. age group was $14 \%$ (16) and $>50$ yrs. was $86 \%$ (98). This indicates, in our study, a significantly high proportion of individuals are in age group 35-49 yrs. In our study the overall prevalence of diabetes was $17.56 \%$ (88 respondents). Prevalence of diabetes among the age groups $<35$ yrs. was $7.9 \%$ ( 7 respondents), $35-49$ yrs. was $55.68 \%$ ( 49 respondents), and $>50$ yrs. was $36.36 \%$ ( 32 respondents). In a similar study conducted by Abhishek A et al., $(2015)^{10}$ in Lucknow, the overall prevalence of diabetes was $15.8 \%$ ( 65 respondents). Age group $<35$ yrs. had $0.7 \%$ ( 1 respondent), $11.4 \%$ (15 respondents), $37.7 \%$ (49 respondents). In contrast to the study conducted by Abhishek A et al. $(2015)^{10}$, in our study, diabetes is more prevalent in the younger age group $<49 \mathrm{yrs}$. (56 out of 88 individuals (63.63\%), compared to 16 out of 65 individuals (24.61\%). Respondents with IDRS $\geq 60$ compared to those with IDRS $<60$ were 4.26 times more at odds of having diabetes after adjusting for other factors. The odds of diabetes among females are 2.23 times compared to males in adjusted model. Zimmet (2003) ${ }^{1}$ indicated excess body fat underlies $64 \%$ of cases of diabetes in men and $77 \%$ of cases in women. Individuals consuming alcohol are 2.06 times more at odds of having diabetes compared to that reporting no to alcohol consumption. Shaw (1987 and 1988$)^{11,12}$ indicated drinking alcohol can contribute to conditions that cause diabetes. Compared to those reporting normal sleep hours (6-8 hrs. per day), respondents with less than $6 \mathrm{hrs}$. sleep duration per day are 2.07 times more at odds of having diabetes. Kristen et al (2007) $)^{13}$ and Karine et al (1999) ${ }^{14}$ also reported reduction in sleep is associated with a significant increase in the incidence of type 2 diabetes. In this study, age, socio-economic status score, habitual smoking, and stress either at home, work or at both places were not significant factors for diabetes in the adjusted model. Prevention measures should aim at reducing and refraining from alcohol, maintaining normal sleep duration of 6-8hrs. per day. In addition, reducing waist circumference through regular physical exercise helps in delaying and/or preventing diabetes. Evaluation of blood glucose levels of the study population would have provided thorough confirmation on their diabetic status.

\section{Conclusion}

In our study, although the distribution of diabetes is more in younger age groups (age < 49 yrs.), age was not a significant factor contributing to diabetes, and all ages are at similar odds for diabetes. Diabetes is not confined to a particular socio-economic status group. Females are more prone to diabetes. Smoking does not affect diabetic status. Alcohol has a significant influence on Diabetes. Sleep duration of $<6 \mathrm{hrs}$. per day has a significant influence on diabetes. 
Normal sleep Duration of 6-8 hrs. per day and beyond (>9 hrs.) are protective to diabetes. However, further research should include dietary aspects including right choice and composition of foods, and timely intake of food.

\section{Acknowledgement}

This paper is the outcome of ICMR studentship project (ICMR STS 2016 Ref. ID: 2016-06991).

\section{Conflict of Interest: None}

\section{References}

1. Zimmet $P$. The burden of type 2 diabetes: are we doing enough? Diabetes Metab 2003; 29(4 Pt 2): 609-618. Available from: https://www.em-consulte.com/en/ article/80233 [DOI: 10.1016/s1262-3636(03)72783-9/ PubMed/ Google Scholar].

2. Geetha M, Kalaivani A, Raja D. Application of Indian diabetes Risk Score in screening of an undiagnosed rural population of Kancheepuram district, Tamil Nadu- A cross sectional survey. NRIMS journal of health sciences 2014; 2(2): 81-83. [Google Scholar].

3. International Diabetes Federation. (Homepage on the internet) IDF diabetes atlas, 6th edition, Brussels, Belgium: International Diabetes Federation 2013. (cited 2014 Jan 17). Available from: http://www.idf.org/ diabetesatlas.

4. Whiting DR, Guaringuata L, Weil C, Shaw J. IDF Diabetes atlas: Global estimates of theprevalence of diabetes for 2011 and 2013. Diabetes Res Clin Pract 2011; 94(3): 311-321. Available from: https://www. diabetesresearchclinicalpractice.com/article/S01688227\%2811\%2900591-2/fulltext [DOI: 10.1016/j. diabres.2011.10.029/ PubMed/ Google Scholar].

5. Ramachandran A, Snehalatha C, Kapur A, Vijay V, Mohan V, Das AK et al. High prevalence of diabetes and impaired glucose tolerance in India: National Urban Diabetes Survey. Diabetologia 2001; 44: 10941101. Available from: https://link.springer.com/ article/10.1007/s001250100627 [DOI: 10.1007/ s001250100627/ PubMed/ Google Scholar].

6. Mohan V, Deepa M, Somannavar S, Datta M. A simplified Indian Diabetic Risk Score for undiagnosed diabetic subjects. J of Association of Physicians in India 2005; 53: 759-763. [PubMed/ Google Scholar].

7. Daniel WW. Biostatistics: A Foundation for analysis in health sciences. $7^{\text {th }}$ edition. New York: John Wiley \& Sons 1999. Available from: https://onlinelibrary.wiley.com/ doi/abs/10.1002/1097-0258\%2820010130\%2920\%3A2 \%3C324\%3A\%3AAID-SIM635\%3E3.0.CO\%3B2-O.

8. Suraj AK, Arunkumar IC, Sumit U, Dipesh P. A study on assessment of risk of Developing diabetes using IDRS (Indian Diabetic Risk Score) in the urban area of Jamnagar city. Scholars Journal of Applied Medical
Sciences SJAMS 2015; 3(6c): 2358-2360. [Google Scholar].

9. Chowdhury R, Mukherjee A, Lahiri SK. A study on distribution and determinants onIndian Diabetic Risk Score (IDRS) among rural population of West Bengal. National Journal of Medical Research 2012; 2(3): 282286. [Google Scholar/ ResearchGate].

10. Abhishek A, Srivastava JP, Pratibha G, Beena S, Daya $\mathrm{P}$, Zeashan HZ. Indian Diabetes Risk Score (IDRS) a strog predictor of diabetes mellitus. A cross sectional study among urban and rural population of Lucknow. International Journal of Applied Research 2015: 1(7): 135-138. Available from: http://www. allresearchjournal.com/archives/2015/vol1issue7/ PartC/1-6-23.1.pdf. [Google Scholar].

11. Shah JH. 'Alcohol decreases insulin sensitivity in healthy subjects'. Alcohol and Alcoholism 1988; 23(2): 103-109. [PubMed/ Google Scholar]

12. Shah J. Alcohol Decreases Insulin Sensitivity In Healthy Subjects" 1988. DOI: http:// dx .doi .org/ 103-109.

13. Knutson KL, Karine Spiegel, Plamen Penev, Eve VaCauter. The Metabolic Consequences of Sleep Deprivation. Sleep Med Rev 2007; 11(3): 163-178. [DOI: 10.1016/j. smrv.2007.01.002/ PubMed/ Google Scholar].

14. Spiegel K, Leproult R, Cauter EV. Impact of sleep debt on metabolic and endocrine function. The Lancet 1999; 354(9188): 1435-1439. [DOI: 10.1016/S01406736(99)01376-8/ PubMed/ Google Scholar]. 